\title{
The Effect of Mutual Coupling on a High Altitude Platform Diversity System Using Compact Antenna Arrays
}

\author{
Tommy Hult ${ }^{1}$ and Abbas Mohammed ${ }^{2}$ \\ ${ }^{1}$ Department of Electrical and Information Technology, Lund University, 22100 Lund, Sweden \\ ${ }^{2}$ Department of Signal Processing, School of Engineering, Blekinge Institute of Technology, 37225 Ronneby, Sweden
}

Correspondence should be addressed to Tommy Hult, tommy.hult@eit.lth.se

Received 30 November 2009; Revised 1 March 2010; Accepted 1 April 2010

Academic Editor: Hon Tat Hui

Copyright ( $\odot 2010$ T. Hult and A. Mohammed. This is an open access article distributed under the Creative Commons Attribution License, which permits unrestricted use, distribution, and reproduction in any medium, provided the original work is properly cited.

\begin{abstract}
We analyze the destructive effects of mutual coupling and spatial correlation between the separate antenna elements on a combined diversity system consisting of multiple HAPs (High-Altitude Platforms) employing various compact MIMO (Multiple-Input Multiple-Output) antenna array configurations, in order to enhance the mutual information in HAP communication links. In addition, we assess the influence of the separation angle between HAPs on system performance, and determine the optimal separation angles that maximize the total mutual information of the system for various compact MIMO antennas. Simulation results show that although the mutual information is degraded by mutual coupling and spatial correlation, the proposed HAP diversity system still provides better performance compared to a nondiversity system for all tested scenarios.
\end{abstract}

\section{Introduction}

High-Altitude Platforms (HAPs) are quasistationary aerial platforms operating in the stratosphere. This emerging technology is preserving many of the advantages of both satellite and terrestrial systems [1-6] and presently started to attract considerable attention in Europe through the European Union COST 297 Action [1]. Using narrow bandwidth repeaters on HAPs for high speed data traffic has several advantages compared to using satellites [1], especially when operating in a local geographical area. In this paper, we propose a multiple HAP diversity system in order to enhance the mutual information in HAP communication links. To further enhance the system performance, independent spatial or polarization channels can be acquired by means of multiple antenna elements at both the transmitter and the receiver, and thus this technique is referred to as Multiple-Input Multiple-Output (MIMO) communications. The MIMO wireless communication is also combined with OFDM (Orthogonal Frequency Division Multiplexing) to form a combined HAP MIMO-OFDM diversity system.

The proposed system consists of virtual MIMO spatial channels (created by multiple HAP diversity) in conjunction with the polarization and antenna pattern diversity (formed via special compact MIMO antenna arrays), as shown in Figure 1. Two compact MIMO antenna array configurations (the MIMO-Cube and MIMO-Tetrahedron) will be investigated, and their performance, in term has of mutual information, is analysed. The enhanced mutual information that is obtained through use of these compact MIMO antenna arrays, and of multiple HAPs, will degrade if the signals of the virtual MIMO spatial channels are correlated. Correlation of the signals can be caused by either low multipath richness in the radio propagation environment or by mutual coupling between the compact antenna array elements. Therefore, the effect of mutual coupling and spatial correlation will be taken into account when performing the simulations for this combined HAP diversity system. We will also analyse the influence of the separation angle between the multiple HAPs on system performance, and determine the optimal separation angles that maximize the total mutual information of the system for the various compact MIMO antennas.

The paper is organized as follows: in Section 2 the proposed combined MIMO-HAP diversity system is introduced. The mathematical analysis of the spatial and polarization channels is presented in Section 3. In Section 4, the various compact MIMO antenna array configurations, 


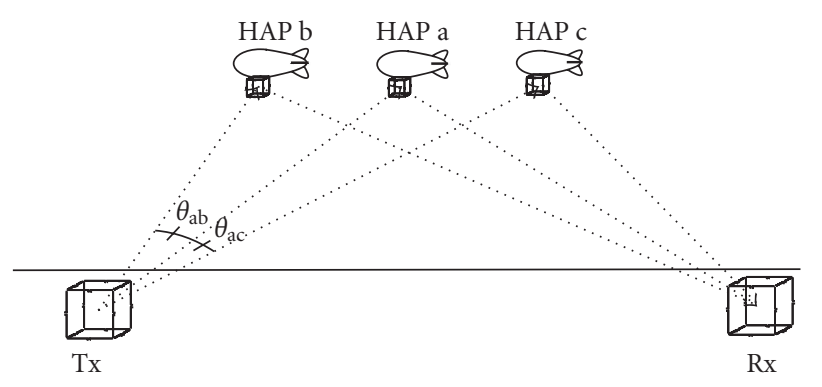

Figure 1: The MIMO-HAP diversity system with three HAPs and the channel paths from the transmitter $(\mathrm{Tx})$ antenna array to the receiver $(\mathrm{Rx})$ antenna array.

their characteristics, and modelling are introduced. The analysis of mutual coupling and spatial correlation effects on these channels is explained in Section 5. Simulation results and comparisons are presented in Section 6. Finally the conclusions are presented in Section 7.

\section{The Proposed Multiple HAP Diversity System}

In this paper, we propose an application for high data rate transmissions using a system employing multiple HAPs. This system consists of a number of uncorrelated MIMO channels created in the space/polarization domain using HAP diversity in combination with a special type of compact MIMO antenna arrays [7-9] as discussed in Section 4. Figure 1 shows the location diversity setup for the case of three HAPs separated by the angle $\theta$.

The propagation channel $\mathbf{H}_{\mathrm{ch}}$ from the transmitter to the receiver can be seen as a simple transformation that contains the distance dependent-free space gain of the signal being transmitted

$$
H_{m n}(\mathbf{r}, f)=\frac{c}{4 \pi f\left|\mathbf{r}_{m}-\mathbf{r}_{n}\right|},
$$

where $\left|\mathbf{r}_{m}-\mathbf{r}_{n}\right|$ is the distance along the path between transmitting antenna element $n$ and receiving antenna element $m$. There are no atmospheric interferences and the noise in the system is modelled as uncorrelated Gaussian noise. The total MIMO channel can then be assembled as

$$
\mathbf{H}=\mathbf{M}_{\mathrm{rx}} \cdot \mathbf{H}_{\mathrm{ch}} \cdot \mathbf{M}_{\mathrm{tx}}
$$

where $\mathbf{M}_{\mathrm{rx}}$ and $\mathbf{M}_{\mathrm{tx}}$ are transformations of the transmitter and receiver antenna signals into and out of the mode domain as described in Section 3.

\section{The Space-Polarization Channel}

The polarization and radiation pattern of the electromagnetic field can be expressed as a multipole expansion [10] of the spherical wave field emanating from a virtual sphere encompassing the antenna array that is being analyzed. This expansion consists of weighted orthogonal vector base functions on the surface of the virtual sphere that allow for a solution to Maxwell's equations in spherical coordinates which can be written as

$$
\begin{aligned}
& \underline{\mathbf{E}}=\sum_{l, m}\left[\frac{j}{k} a_{E}(l, m)\left(\nabla \times f_{l}(k r)\right) \underline{\mathbf{X}}_{l m}+a_{M}(l, m) g_{l}(k r) \underline{\mathbf{X}}_{l m}\right], \\
& \underline{\mathbf{H}}=\frac{1}{\eta} \sum_{l, m}\left[a_{E}(l, m) f_{l}(k r) \underline{\mathbf{X}}_{l m}-\frac{j}{k} a_{M}(l, m)\left(\nabla \times g_{l}(k r)\right) \underline{\mathbf{X}}_{l m}\right],
\end{aligned}
$$

where $\eta$ is the intrinsic impedance of vacuum and underscoring denotes a field vector. The base functions $\underline{\mathbf{X}}_{l m}(\varphi, \theta)$ represent mode $(l, m)$ of the electromagnetic field in spherical coordinates when the far-field of the antenna is projected onto the virtual sphere. The functions $g_{l}$ and $f_{l}$ in (3) are spherical Hankel functions of order $l$ representing an outgoing (transmitted) or incoming (received) wave. The scalar weights $a_{E}$ and $a_{M}$ are the corresponding coefficients which will give the gain of each orthogonal function (mode) for a particular electromagnetic far-field pattern, as shown by

$$
\begin{gathered}
a_{E}(l, m) \approx \frac{1}{\eta_{0}} \cdot \frac{k}{\sqrt{l(l+1)}} f_{l}(k r) \int Y_{l m}^{*}(\theta, \varphi) \mathbf{r} \cdot \mathbf{E} d^{3} \mathbf{r} \\
a_{M}(l, m) \approx-\frac{k}{\sqrt{l(l+1)}} g_{l}(k r) \int Y_{l m}^{*}(\theta, \varphi) \mathbf{r} \cdot \mathbf{H} d^{3} \mathbf{r} .
\end{gathered}
$$

Using (4) we can calculate which modes are active on any arbitrary antenna enveloped by the virtual sphere by knowing the electric and magnetic field patterns of the antenna. These modes are theoretically orthogonal to each other and therefore represent independent ports of the antenna. The transmitting antenna-channel transform matrix $\mathbf{M}_{\mathrm{tx}}$ is then assumed to be the linear transformation of the input signals $\mathbf{x}$ into the mode domain $\mathbf{a}_{\mathrm{tx}}$ according to $\mathbf{a}_{\mathrm{tx}}=\mathbf{M}_{\mathrm{tx}} \mathbf{x}$, and for the receiving channel-antenna, we have a similar transformation matrix from the mode domain $\mathbf{a}_{\mathrm{rx}}$ into the output signals $\mathbf{y}$ of the system following $\mathbf{y}=\mathbf{M}_{\mathrm{rx}} \mathbf{a}_{\mathrm{rx}}$, where $\mathbf{a}_{\mathrm{tx}}$ and $\mathbf{a}_{\mathrm{rx}}$ are vectors containing the mode gains for $\mathrm{a}$ specific antenna type.

\section{The Compact MIMO Antenna Array Configurations}

Each transmit and receive antenna arrangement of the proposed system consists of a special compact MIMO antenna array configuration, which possesses a structure of different designs and complexities as shown in Figure 2.

The first compact MIMO antenna array is known as the MIMO-Cube [10], which consists of twelve electric dipoles positioned on the twelve edges of a cube. It can be seen from Figure 3 that this antenna array theoretically has twelve independent antenna ports.

The second compact antenna that we have investigated is known as the MIMO-Tetrahedron, which has six electric dipoles placed at the six edges of a tetrahedron (see Figure 2), and so theoretically has six independent ports as can be seen in Figure 4. 


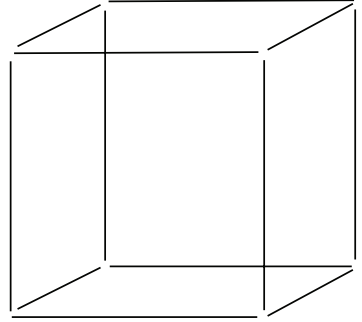

(a)

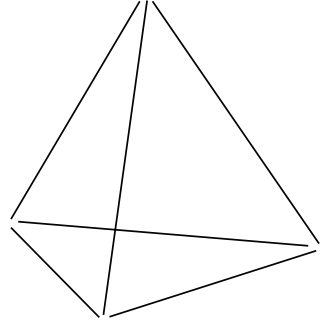

(b)
FIgURE 2: The structure of the two compact MIMO antenna array configurations: (a) MIMO-cube and (b) MIMO-tetrahedron.

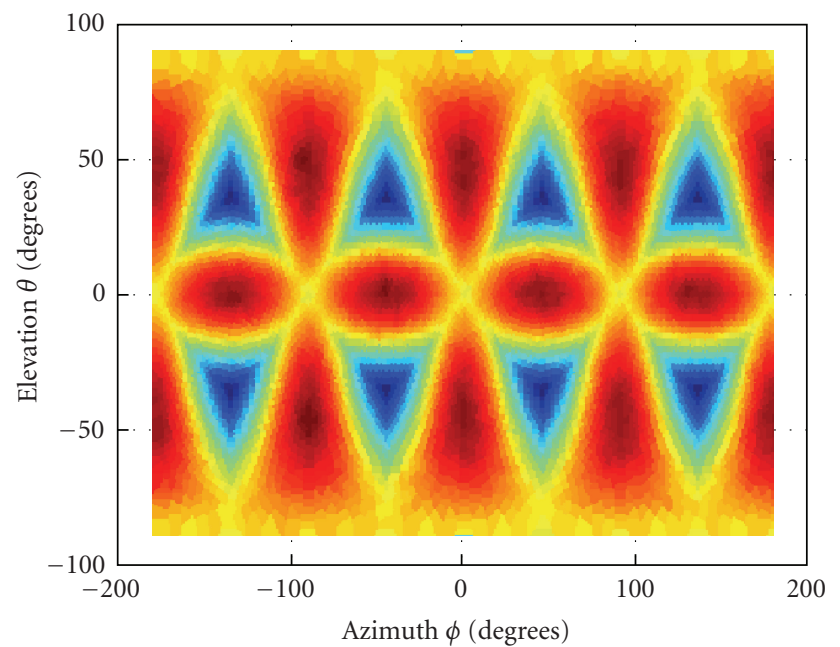

FIGURE 3: The theoretical antenna pattern when all twelve channels of the MIMO-Cube antenna are activated. The four antenna beams at elevation angle 0 degrees have vertical linear polarization and the eight beams at elevation \pm 45 degrees have horizontal linear polarization.

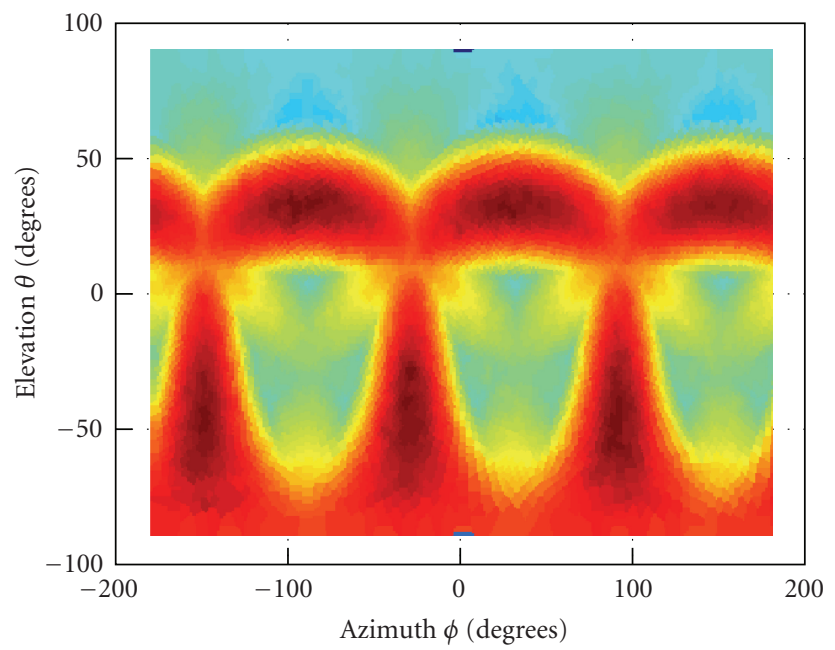

FIGURE 4: The theoretical antenna pattern when all six channels of the MIMO-Tetrahedron antenna are activated. The three antenna beams at elevation angle 30 degrees have vertical linear polarization and the three beams at elevation -45 degrees have horizontal linear polarization.
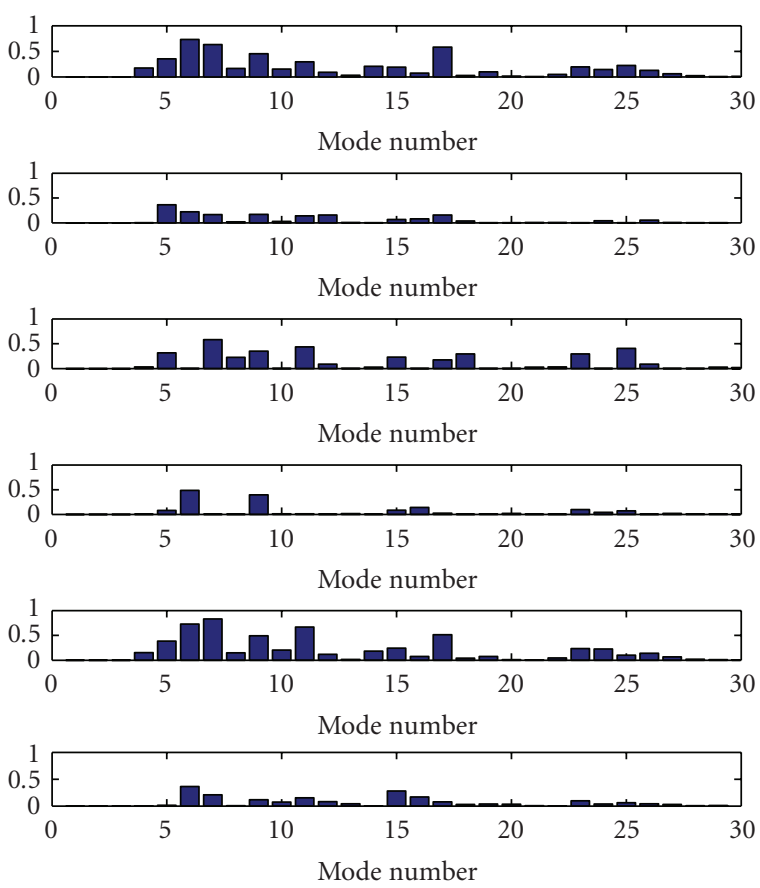

FIGURE 5: The activated modes of the MIMO-Tetrahedron antenna array. Each bar-graph represents the modes activated on each of the six antenna array elements.

In Figure 5 we show an example of the activation of the first 30 modes calculated by (4) for the MIMO-Tetrahedron antenna array.

In order to separate the modes in Figure 5 into independent MIMO channels, we employ a singular value decomposition (SVD) operation

$$
\mathbf{H}=\mathbf{U} \Sigma \mathbf{V}^{\mathrm{H}},
$$

where $\mathbf{U}$ and $\mathbf{V}$ are unitary eigenvector matrices of the MIMO channel modes and $\Sigma$ comprises the subchannel gains of the independent ports of the antenna array.

The orthogonalization of the channel into the independent subchannels is accomplished by multiplying the signal to be transmitted $\mathbf{x}$ with the matrix $\mathbf{V}$ on the transmitter side of the channel, and multiply the received signal $\mathbf{y}$ on the receiver side of the channel with the matrix $\mathrm{U}^{\mathrm{H}}$ according to

$$
\mathbf{y}=\mathbf{U}^{\mathrm{H}} \mathbf{H}(\mathbf{V} \mathbf{x})+\mathbf{U}^{\mathrm{H}} \mathbf{v}
$$

where $\mathbf{y}$ is a vector containing the received and decoded signal from the independent MIMO subchannels. Inserting (5) into (6) then orthogonalizes the channels into

$$
\mathbf{y}=\Sigma \mathbf{x}+\mathbf{w},
$$

where $\mathbf{w}=\mathbf{U}^{\mathrm{H}} \mathbf{v}$ is the received noise. Figure 6 shows the orthogonalized subchannels for the MIMO-Cube and MIMO-Tetrahedron antenna arrays, respectively. It can be clearly seen from this figure that the MIMO-Cube antenna provides superior channel gain levels compared to the MIMO-Tetrahedron antenna. This translates to higher 


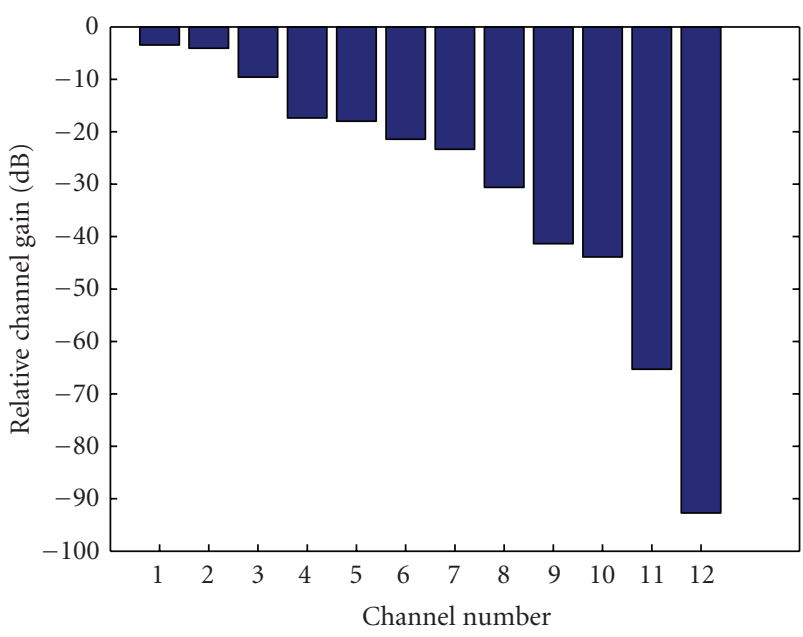

(a)

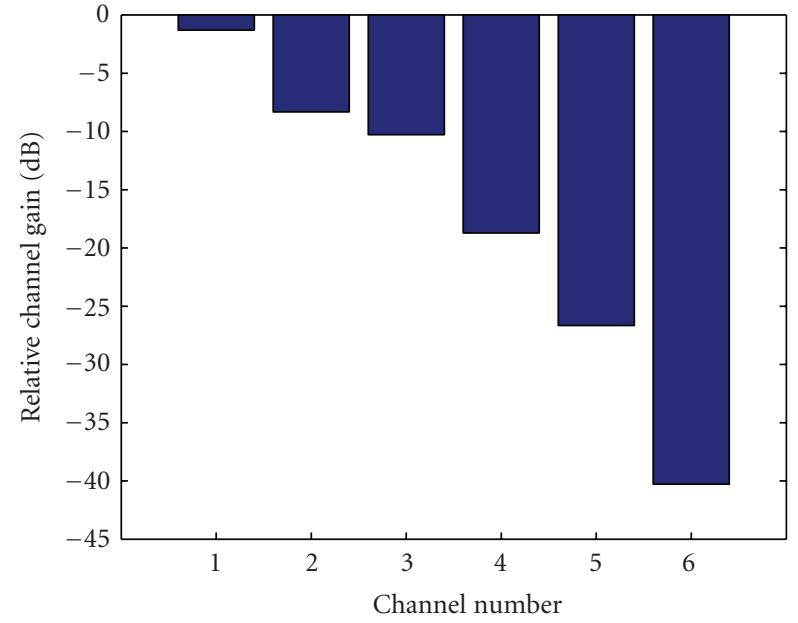

(b)

FIGURE 6: The orthogonalized 12 subchannels of the MIMO-Cube antenna (a), and the 6 subchannels of the MIMO-Tetrahedron antenna (b).

system mutual information as will be shown in the results section.

Through the combination MIMO-OFDM (Orthogonal Frequency Division Multiplexing), we also achieve orthogonality over the frequency domain by using the Discrete Fourier Transform (DFT) with a Cyclic Prefix (CP). Assuming a cyclic prefix $\mathrm{CP}>\tau_{d} \cdot f_{s}$, where $f_{s}=1 / T_{s}$ and $T_{s}$ is the symbol time, we can write the received signals as a circular convolution over the DFT-frame

$$
r_{m}(t)=\sum_{n=1}^{N} h_{m n}(t) \otimes s_{n}(t)+v_{m}(t), \quad t \in\left[0, \frac{K-1}{f_{s}}\right],
$$

where $K$ is the size of the DFT, $m=\{1, \ldots, M\}$ is receiving antennas, $n=\{1, \ldots, N\}$ is transmitting antennas, and $v_{m}(t)$ is AWGN. If we have a MIMO antenna system with $N$ transmitting antennas and $M$ receiving antennas, we can then write the signal for subchannel $k$ in the frequency domain between any pair of transmitting and receiving antennas as

$$
R_{m}(k)=\sum_{n=1}^{N} H_{m n}(k) S_{n}(k)+W_{m n}(k), \quad k \in[0, K-1],
$$

where $k$ denotes each separate subcarrier. If we write (9) in vector notation, we get

$$
\mathbf{R}(k)=\mathbf{H}(k) \mathbf{S}(k)+\mathbf{W}(k),
$$

where $\mathbf{R}(k)$ is an $M \times 1$ vector and $\mathbf{S}(k)$ is an $N \times 1$ vector of the received and transmitted signals, respectively. $\mathbf{H}(k)$ is the $M \times N$ frequency response matrix of the channel between $N$ transmitters and $M$ receivers. The noise in the system $\mathbf{W}(k)$ is an $M \times 1$ vector assumed to be additive white Gaussian noise. The correlation matrix of the noise vector $\mathbf{W}(k)$ is thereby $E\left\{\mathbf{W}(k) \mathbf{W}^{\mathrm{H}}(\mathrm{k})\right\}=\sigma_{n}^{2} \cdot \mathbf{I}_{M}$, where $\sigma_{n}^{2}$ is the variance of the noise and $\mathbf{I}_{M}$ is the $M \times M$ identity matrix. Since we are using singular value decomposition (SVD) technique, we can now write the channel frequency response matrix $\mathbf{H}(k)$ as

$$
\mathbf{H}(k)=\mathbf{U}(k) \Sigma(k) \mathbf{V}^{\mathbf{H}}(k),
$$

where $\Sigma(k)$ is an $M \times N$ matrix containing singular values that are larger than zero $\sigma_{1}(k) \geq \sigma_{2}(k) \geq \cdots \geq \sigma_{r}(k)>0$, where $r$ is the rank of the matrix $\mathbf{H}(k)$, and the $M \times M$ matrix $\mathbf{U}(k)$ and the $N \times N$ matrix $\mathbf{V}(k)$ are unitary and contain the corresponding eigenvectors as matrix column vectors. To obtain a diagonalized system, we then define

$$
\mathbf{Y}(k)=\Sigma(k) \mathbf{X}(k)+\mathbf{N}(k)
$$

where

$$
\begin{gathered}
\mathbf{Y}(k)=\mathbf{U}^{\mathbf{H}}(k) \mathbf{R}(k), \\
\mathbf{S}(k)=\mathbf{V}(k) \mathbf{X}(k), \\
\mathbf{N}(k)=\mathbf{U}^{\mathbf{H}}(k) \mathbf{W}(k) .
\end{gathered}
$$

Since the MIMO-OFDM channels in (12) are uncorrelated and the correlation of the noise $\mathbf{N}(k)$ is $E\left\{\mathbf{N}(k) \mathbf{N}^{\mathrm{H}}(k)\right\}=\sigma_{n}^{2}$. $\mathbf{I}_{M}$, then we can write the mutual information of the system [11] as

$$
C=\sum_{k=0}^{K-1} \sum_{m=1}^{r} \log _{2}\left(1+\sigma_{x_{m}}^{2}(k) \frac{\sigma_{m}^{2}(k)}{\sigma_{n}^{2}}\right),
$$

where $\sigma_{x_{m}}^{2}(k)$ is the variance of the separate uncorrelated input signals in vector $\mathbf{X}(k)$ and $r$ is the rank of the system. The mutual information in (14) is constrained by the total radiated power from the transmitting antennas, defined as

$$
P_{\mathrm{rad}}=\sum_{k=0}^{K-1} \sum_{m=1}^{r} \sigma_{x_{m}}^{2}(k)
$$

To maximize the total sum of mutual information in all the subchannels, we use the so-called "water-filling" technique in which we allocate more power to the subchannels 


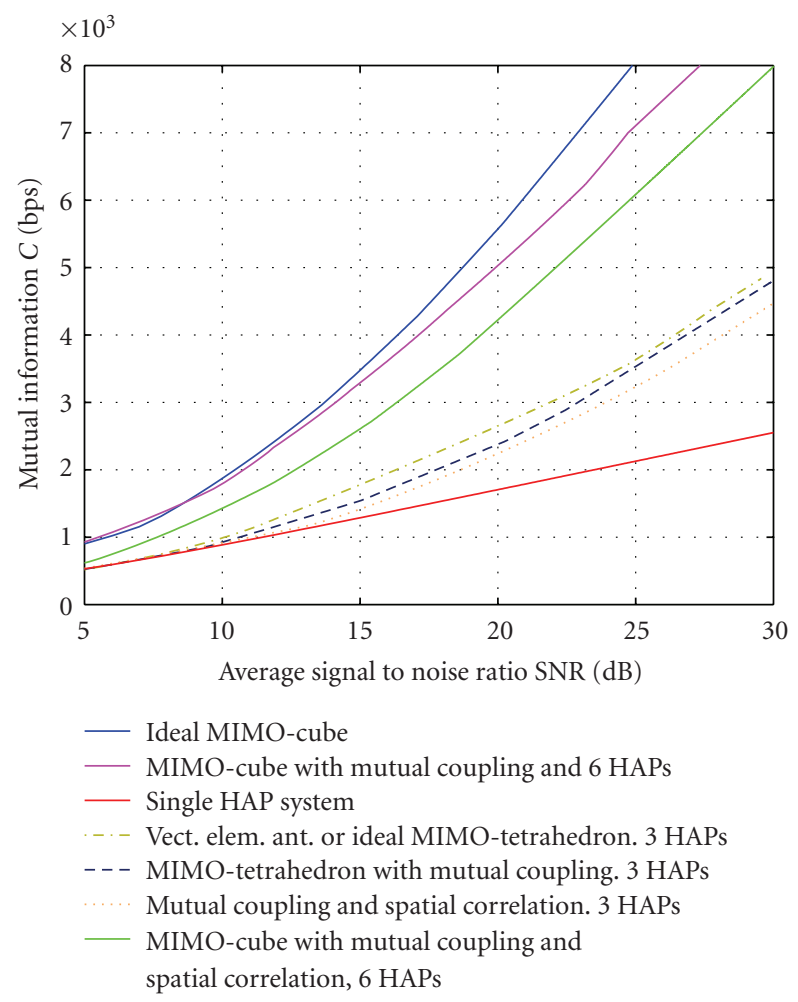

FIgURE 7: The mutual information versus average SNR for different configurations of the MIMO-Cube and MIMO-Tetrahedron antenna arrays in comparison to the ideal vector element antenna array and a single HAP system. The elevation angle is 10 degrees and the separation angle between HAPs is 20 degrees.

with high eigenvalues. The optimal "water-filling" solution $[12]$ is then given by

$$
\sigma_{x_{m}}^{2}(k)= \begin{cases}\gamma-\frac{\sigma_{n}^{2}}{\sigma_{m}^{2}(k)} & \text { if } \gamma-\frac{\sigma_{n}^{2}}{\sigma_{m}^{2}(k)}>0, \\ 0 & \text { if } \gamma-\frac{\sigma_{n}^{2}}{\sigma_{m}^{2}(k)}>0,\end{cases}
$$

where $k=\{0, \ldots, K-1\}, m=\{1, \ldots, r\}, \sigma_{n}^{2}$ is the variance of the noise, and $\gamma$ is a predefined threshold level of the signal-to-noise ratio in the system and it is dependent on the total transmitted power $P_{\text {rad }}$ in (15). The average signal-tonoise ratio $\mathrm{SNR}_{\mathrm{avg}}$ in the simulations is calculated as

$$
\mathrm{SNR}_{\mathrm{avg}}=10 \log _{10}\left(\frac{1}{N_{\mathrm{active}}} \sum_{k=0}^{K-1} \sum_{m=1}^{r} \sigma_{x_{m}}^{2}(k) \cdot \frac{\sigma_{m}^{2}(k)}{\sigma_{n}^{2}}\right),
$$

where $N_{\text {active }}$ is the number of active channels used for which the variance of the input signals $\sigma_{x_{m}}^{2}(k)>0$.

\section{Mutual Coupling and Spatial Correlation}

The interaction between the elements of the compact antenna array will cause mutual interference in the radiation patterns of the array elements, and this effect is known as "mutual coupling" [13-17]. The mutual coupling between the elements of the compact antenna array can be viewed as a multiport network on the transmitter and receiver sides, respectively, in which the relationships between the terminal voltages and currents of the different ports can be define as [13],

$$
\begin{aligned}
& \mathbf{u}_{\mathrm{tx}}=\mathbf{Z}_{\mathrm{tx}} \mathbf{i}_{\mathrm{tx}}, \\
& \mathbf{u}_{\mathrm{rx}}=\mathbf{Z}_{\mathrm{rx}} \mathbf{i}_{\mathrm{rX}},
\end{aligned}
$$

where $\mathbf{Z}_{\mathrm{tx}}$ and $\mathbf{Z}_{\mathrm{rx}}$ are the impedance matrices of the antenna array. $\mathbf{Z}_{\mathrm{tx}}$ and $\mathbf{Z}_{\mathrm{rx}}$ have been calculated numerically by using the finite element method (FEM) for the antenna arrays shown in Figure 2. An infinite approximation of the array is assumed by not taking the edge effects of the finite array into account. The terminal voltages are then associated with the source voltages through the source impedances $Z_{S}$, as described in [13],

$$
\begin{aligned}
& \mathbf{u}_{\mathrm{tx}}=\mathbf{Z}_{\mathrm{TX}} \mathbf{u}_{S}^{(\mathrm{TX})}, \\
& \mathbf{u}_{\mathrm{rX}}=\mathbf{Z}_{\mathrm{RX}} \mathbf{u}_{S}^{(\mathrm{RX})},
\end{aligned}
$$

where $\mathbf{u}_{S}^{(\mathrm{TX})}$ is the source voltage at the transmitter and $\mathbf{u}_{S}^{(\mathrm{RX})}$ is the source voltage at the receiver generated by the impinging electromagnetic wave. The impedance coupling matrices are denoted as $\mathbf{Z}_{\mathrm{TX}}$ and $\mathbf{Z}_{\mathrm{RX}}$, and are defined by

$$
\begin{aligned}
& \mathbf{Z}_{\mathrm{TX}}=\frac{\mathbf{Z}_{\mathrm{tx}}\left(\mathbf{Z}_{\mathrm{tx}}+\mathbf{Z}_{S}\right)^{-1}}{C_{\mathrm{tx}}}, \\
& \mathbf{Z}_{\mathrm{RX}}=\frac{\mathbf{Z}_{L}\left(\mathbf{Z}_{\mathrm{rX}}+\mathbf{Z}_{L}\right)^{-1}}{C_{\mathrm{rx}}},
\end{aligned}
$$

where $\mathbf{Z}_{\mathrm{tx}}$ and $\mathbf{Z}_{\mathrm{rx}}$ are the impedance matrices of the transmitting and receiving compact antenna arrays, $\mathbf{Z}_{S}$ and $\mathbf{Z}_{L}$ are diagonal matrices containing the source and load impedances which have been chosen as the complex conjugate of the self-impedance of the antenna given by the diagonal $\left[\mathbf{Z}_{\mathrm{tx}}\right]_{i i}$ and $\left[\mathbf{Z}_{\mathrm{rx}}\right]_{i i}$ of the impedance matrices $\mathbf{Z}_{\mathrm{tx}}$ and $\mathbf{Z}_{\mathrm{rx}}$, and $C_{\mathrm{tx}}=\left[\mathbf{Z}_{\mathrm{tx}}\right]_{11} /\left(\left[\mathbf{Z}_{\mathrm{tx}}\right]_{11}+\left[\mathbf{Z}_{\mathrm{tx}}^{*}\right]_{11}\right)$ and $C_{\mathrm{rx}}=$ $\left[\mathbf{Z}_{\mathrm{rx}}\right]_{11} /\left(\left[\mathbf{Z}_{\mathrm{rx}}\right]_{11}+\left[\mathbf{Z}_{\mathrm{rx}}^{*}\right]_{11}\right)$ are normalizing factors $[13]$.

In (8) it was shown that the complex envelope of the received signal at the receiving compact array after matched filtering can be expressed in vector notation as

$$
\mathbf{r}=\mathbf{H s}+\mathbf{v}
$$

where $\mathbf{s}$ is the $N \times 1$ vector containing the signals from the $N$ transmitting antenna elements, $\mathbf{v}$ is the $M \times 1$ zero-mean AWGN vector, and $\mathbf{H}$ is the normalized $M \times N$ channel matrix that, assuming separable transmit and receive spatial correlation matrices, is modeled using the Kronecker model $[18,19]$,

$$
\mathbf{H}=\mathbf{Z}_{\mathrm{RX}}\left(\mathbf{R}_{\mathrm{rX}}\right)^{1 / 2} \mathbf{H}_{\mathbf{0}}\left(\mathbf{R}_{\mathrm{tX}}\right)^{1 / 2} \mathbf{Z}_{\mathrm{TX}},
$$

where $\mathbf{H}_{\mathbf{0}}$ is an i.i.d. channel response without spatial correlation and mutual coupling. $\mathbf{R}_{\mathrm{rx}}$ and $\mathbf{R}_{\mathrm{tx}}$ are the separable spatial correlation matrices on the receiving and transmitting sides, respectively. 


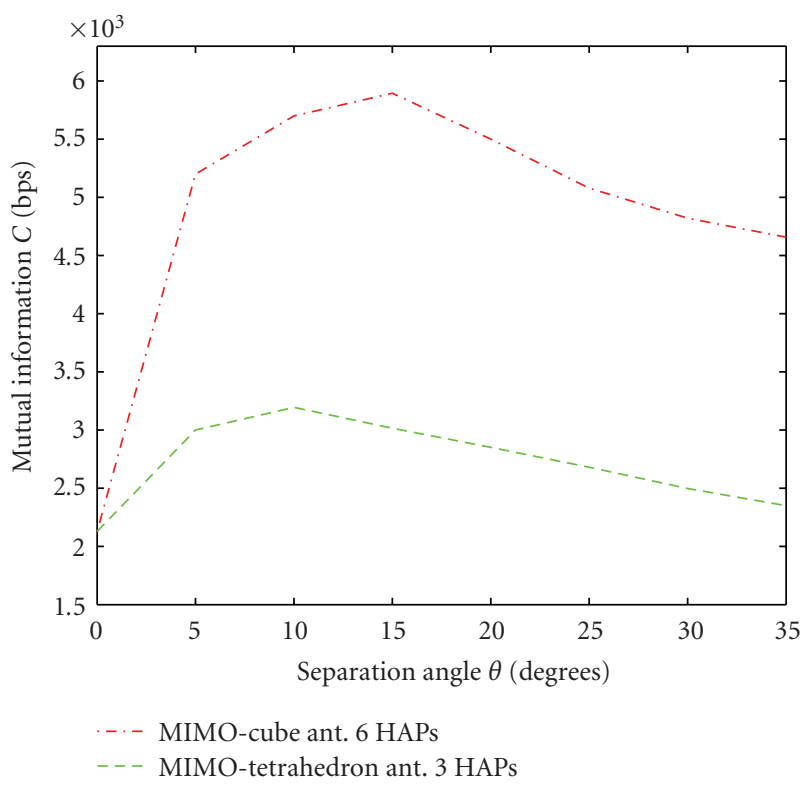

Figure 8: The total system mutual information versus separation angle between HAPs for an average SNR of $20 \mathrm{~dB}$ for the two compact MIMO antenna array configurations.

The spatial correlation matrices $\mathbf{R}_{\mathrm{tx}}$ and $\mathbf{R}_{\mathrm{rx}}$ are calculated between the antenna element positions and polarization states according to [20]

$$
\begin{aligned}
\rho_{p, q}= & \frac{\int_{\varphi} \int_{\theta} \int_{\zeta} \int_{\mathcal{K}} \boldsymbol{\alpha}_{q}(\Theta) \boldsymbol{\alpha}_{p}^{*}(\boldsymbol{\Theta}) \sin (\theta) \mathbf{p}(\boldsymbol{\Theta}) d \kappa d \zeta d \theta d \varphi}{\sqrt{\int_{\varphi} \int_{\theta} \int_{\zeta} \int_{\mathcal{K}}\left|\boldsymbol{\alpha}_{q}(\boldsymbol{\Theta})\right|^{2} \sin (\theta) \mathbf{p}(\boldsymbol{\Theta}) d \kappa d \zeta d \theta d \varphi}} \\
& \times \frac{1}{\sqrt{\int_{\varphi} \int_{\theta} \int_{\zeta} \int_{\mathcal{K}}\left|\boldsymbol{\alpha}_{p}(\boldsymbol{\Theta})\right|^{2} \sin (\theta) \mathbf{p}(\boldsymbol{\Theta}) d \kappa d \zeta d \theta d \varphi}},
\end{aligned}
$$

where element $\rho_{p, q}$ of matrix $\mathbf{R}_{\mathrm{tx}}$ or $\mathbf{R}_{\mathrm{rx}}$ is the spatial correlation between antenna elements $p$ and $q \cdot \varphi$ and $\theta$ are the spherical coordinates expressing the spatial domain and $\zeta$ and $\kappa$ are the polarization angle and phase difference, respectively, that account for the effects in the polarization domain [20]. In (23), $\boldsymbol{\alpha}(\boldsymbol{\Theta})$ is the steering vector and $\mathbf{p}(\boldsymbol{\Theta})$ is the joint probability distribution function of the parameter vector $\Theta=\left[\begin{array}{llll}\theta & \varphi & \zeta & \kappa\end{array}\right]^{\mathrm{T}}$, where $\mathrm{T}$ denotes a transpose operator. It is assumed that all the parameters are independent and that $p(\zeta)=u\{0, \pi\}$ and $p(\kappa)=u\{-\pi, \pi\}$ are uniformly distributed.

\section{Simulation Results}

In this section, we investigate the mutual information improvement resulting from the use of multiple HAPs system employing compact MIMO antenna arrays and compare the results to that obtained from a system without diversity. In addition, we will also show the effects of mutual coupling and spatial-polarization correlation (calculated by the Finite Element Method) on the mutual information of the system. The mutual information results shown in Figure 7 are obtained for a system of HAPs operating at an altitude of $22 \mathrm{~km}$ and with a separation angle of 20 degrees, and plotted against the average signal-to-noise ratio of the system. The elevation angle is held at 10 degrees throughout the simulations.

It is evident from Figure 7 that the HAP diversity system provides superior performance as compared to the single HAP case, and the MIMO-Cube antenna provides more mutual information than the MIMO-Tetrahedron antenna which is the result of the higher number of acquired HAP platforms. Simulation results also show that although the mutual information is degraded by correlation and mutual coupling, we still achieve significant gain compared to the single HAP case. From Figure 7 it is also clear that for example, for an average SNR of $20 \mathrm{~dB}$ we get a $110 \%$ more mutual information from the ideal MIMO-Cube antenna than from the ideal MIMO-Tetrahedron antenna, and a $228 \%$ more mutual information compared with the nondiversity single HAP system. If we take into account the correlation and the mutual coupling, the MIMO-Cube antenna still has a $108 \%$ more mutual information than the MIMO-Tetrahedron antenna and a 195\% more mutual information than the ideal nondiversity single HAP system.

In Figure 8, we show the effect of separation angles between HAPs on the mutual information of the two compact MIMO antenna arrays. The separation angle $\theta$ (see Figure 2) is varied from 0 to 35 degrees in steps of 5 degrees (due to computational limitations) and plotted for an average SNR of $20 \mathrm{~dB}$. It is clear from this figure that the separation angle between HAPs has a great impact on the mutual information of the system. It is also evident that the optimal separation angle that maximizes the total mutual information of the system at this SNR is found to be approximately 10 degrees for the MIMO-Tetrahedron antenna and approximately 15 degrees for the MIMO-Cube antenna. The degradation of mutual information performance at wider separation angles is due to the increase in distance between the HAPs and the ground stations. Figure 8 also suggests that the vector element antenna array is superior to the MIMOTetrahedron array at wide separation angles even though they access the same number of independent subchannels.

\section{Conclusions}

In this paper, we have investigated the degradation in mutual information, due to spatial correlation and mutual coupling, when using different compact MIMO antenna arrays in a multiple HAP system employing multiple spatialpolarization modes. Simulation results show that the multiple HAP diversity systems utilizing compact MIMO antenna arrays provide significant mutual information enhancement, even though it can also be noticed that small degradation in mutual information is resulted due to the effects of spatial correlation and mutual coupling between the separate antenna elements of the MIMO-Cube and MIMOTetrahedron arrays. It was also shown that the performance of the compact MIMO-Cube antenna is superior to the MIMO-Tetrahedron antenna since it has twice the number 
of independent channels which will result in more system mutual information. Finally, we have also shown the effects of the separation angle between HAPs on system performance, and determined that the optimal separation angle that maximizes the total mutual information of the system is within a narrow sector of approximately 20 degrees for the compact MIMO antenna arrays investigated in this paper.

\section{References}

[1] A. Mohammed, S. Arnon, D. Grace, M. Mondin, and R. Miura, "Advanced communication techniques and applications for high-altitude platforms," EURASIP Journal on Wireless Communications and Networking, vol. 2008, Article ID 934837, 3 pages, 2008.

[2] T. Hult, D. Grace, and A. Mohammed, "WCDMA uplink interference assessment from multiple high altitude platform configurations," EURASIP Journal on Wireless Communications and Networking, vol. 2008, Article ID 182042, 7 pages, 2008.

[3] A. Widiawan, R. Tafazolli, B. Evans, V. Milas, and P. Constantinou, "Coexistence of high altitude platform station, satellite, and terrestial systems for fixed and mobile services," in Proceedings of International Workshop on High Altitude Platform Systems, Athens, Greece, September 2005.

[4] V. F. Milas and P. Constantinou, "Interference environment between high altitude platform networks (HAPN), geostationary (GEO) satellite and wireless terrestrial systems," Wireless Personal Communications, vol. 32, no. 3-4, pp. 257-274, 2005.

[5] G. Aranti, A. Iera, and A. Molinaro, "The role of HAPs in supporting multimedia broadcast and multicast services in terrestrial-satellite integrated systems," Wireless Personal Communications, vol. 32, no. 3-4, pp. 195-213, 2005.

[6] G. Chen, D. Grace, and T. C. Tozer, "Performance of multiple high altitude platforms using directive HAP and user antennas," Wireless Personal Communications, vol. 32, no. 3-4, pp. 275-299, 2005.

[7] S. Nordebo, A. Mohammed, and J. Lundbäck, "On the use of polarization channels in satellite communications," in Proceeding of Radio Vetenskap och Kommunication, Stockholm, Sweden, June 2002.

[8] M. R. Andrews, P. P. Mitra, and R. Decarvalho, "Tripling the capacity of wireless communications using electromagnetic polarization," Nature, vol. 409, no. 6818, pp. 316-318, 2001.

[9] J. Andersen and B. Getu, "The MIMO cube-a compact MIMO antenna," in Proceedings of the 5th IEEE International Symposium on Wireless Personal Multimedia Communications, vol. 1, pp. 112-114, 2002.

[10] R. Fitzpatrick, Lecture Notes: Advanced Classical Electromagnetism, University of Texas, Austin, Tex, USA, 1996.

[11] M. A. Khalighi, J.-M. Brossier, G. Jourdain, and K. Raoof, "Water filling capacity of Rayleigh MIMO channels," in Proceedings of the 12th IEEE International Symposium on Personal, Indoor and Mobile Radio Communications (PIMRC '01), vol. 1, pp. A155-A158, San Diego, Calif, USA, September 2001.

[12] G. J. Foschini and M. J. Gans, "On limits of wireless communications in a fading environment when using multiple antennas," Wireless Personal Communications, vol. 6, no. 3, pp. 311-335, 1998.

[13] R. Janaswamy, "Effect of element mutual coupling on the capacity of fixed length linear arrays," IEEE Antennas and Wireless Propagation Letters, vol. 1, pp. 157-160, 2002.
[14] C. Waldschmidt, S. Schulteis, and W. Wiesbeck, "Complete RF system model for analysis of compact MIMO arrays," IEEE Transactions on Vehicular Technology, vol. 53, no. 3, pp. 579586, 2004.

[15] J. W. Wallace and M. A. Jensen, "Mutual coupling in MIMO wireless systems: a rigorous network theory analysis," IEEE Transactions on Wireless Communications, vol. 3, no. 4, pp. 1317-1325, 2004.

[16] Y. Yang, R. S. Blum, and S. Sfar, "Antenna selection for MIMO systems with closely spaced antennas," EURASIP Journal on Wireless Communications and Networking, vol. 2009, Article ID 739828, 11 pages, 2009.

[17] T. Svantesson and A. Ranheim, "Mutual coupling effects on the capacity of multielement antenna systems," in Proceedings of IEEE International Conference on Acoustics, Speech and Signal Processing (ICASSP '01), vol. 4, pp. 2485-2488, Salt Lake City, Utah, USA, May 2001.

[18] D.-S. Shiu, G. J. Foschini, M. J. Gans, and J. M. Kahn, "Fading correlation and its effect on the capacity of multielement antenna systems," IEEE Transactions on Communications, vol. 48, no. 3, pp. 502-513, 2000.

[19] J. P. Kermoal, L. Schumacher, K. I. Pedersen, P. E. Mogensen, and F. Frederiksen, "A stochastic MIMO radio channel model with experimental validation," IEEE Journal on Selected Areas in Communications, vol. 20, no. 6, pp. 1211-1226, 2002.

[20] S. K. Yong and J. S. Thompson, "Three-dimensional spatial fading correlation models for compact MIMO receivers," IEEE Transactions on Wireless Communications, vol. 4, no. 6, pp. 2856-2868, 2005. 

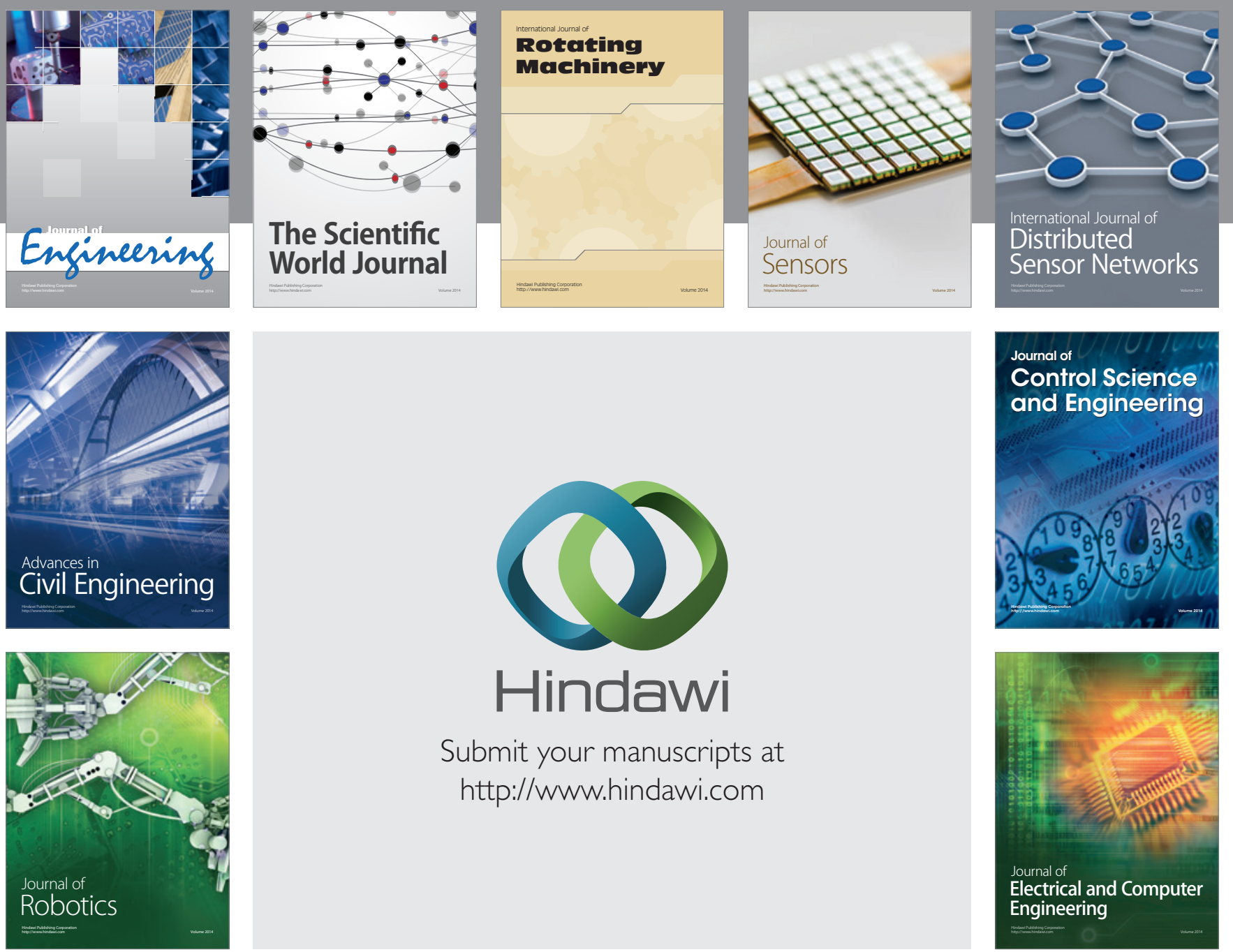

Submit your manuscripts at

http://www.hindawi.com
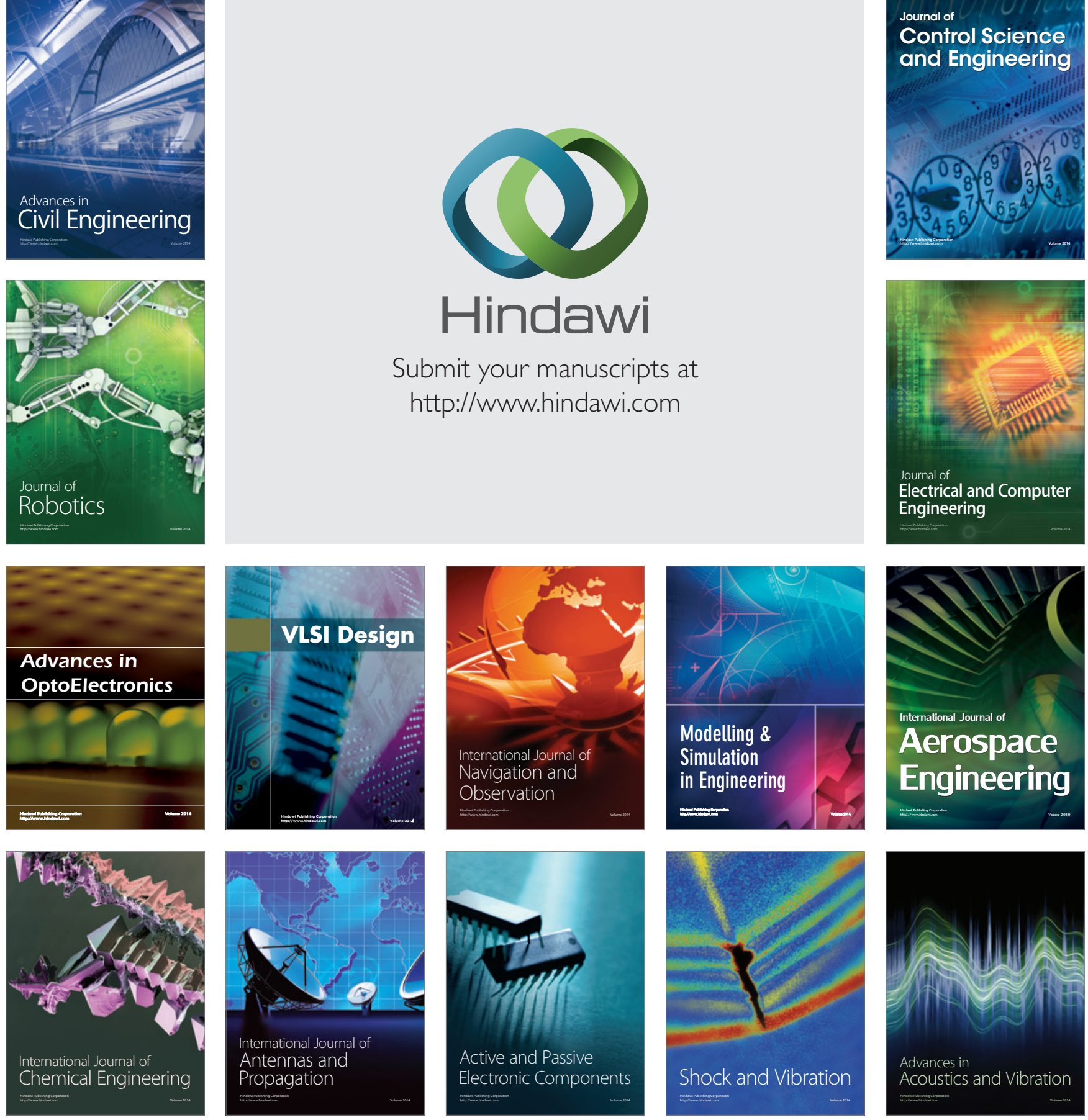